\title{
Adaptação Portuguesa da Escala de Qualidade de Vida para Pessoas com Esquizofrenia
}

\author{
Portuguese Adaptation of the Quality of Life Scale for Schizophrenic Patients
}

\author{
Lara Guedes de Pinho ${ }^{1}$, Anabela Pereira ${ }^{2}$ e Cláudia Chaves ${ }^{3}$
}

\begin{abstract}
Resumo
A esquizofrenia é uma doença mental grave e crónica que acomete a qualidade de vida de quem dela padece. A Quality of Life Scale (QLS), de Heinrichs, Hanlon e Carpenter, 1984, é uma das escalas específicas mais utilizadas a nível internacional para a avaliar. Por não existir em Portugal nenhum instrumento ajustado para a esquizofrenia, este estudo tem como objetivo proceder à adaptação e validação para a população portuguesa desta escala, na sua versão reduzida, com 7 itens. A amostra foi constituída por 282 participantes com diagnóstico de esquizofrenia de diferentes instituições do país. A análise das qualidades psicométricas (análise fatorial exploratória e consistência interna) do instrumento avaliado mostrou índices adequados de fidedignidade e validade, consistentes com os dados internacionais existentes. Estes resultados justificam a utilização do mesmo no contexto português.
\end{abstract}

Palavras-chave: esquizofrenia, qualidade de vida, escala, avaliação, Quality of Life Scale (QLS)

\begin{abstract}
Schizophrenia is a severe and chronic mental illness that affects the quality of life of those who suffer from it. The Quality of Life Scale (QLS), by Heinrichs, Hanlon and Carpenter, 1984, is one of the most specific scales used internationally to assess it. Because in Portugal there is no instrument designed for schizophrenia, the aim of this study is to adapt and validate this scale for the Portuguese population in its reduced 7-item version. The sample consisted of 282 participants with schizophrenia in different health institutions from north to south in the country. Analysis of the instrument's psychometric properties (exploratory factor analysis and internal consistency) showed adequate levels of reliability and validity consistent with existing international data. These results justify the use of this scale in the Portuguese context.
\end{abstract}

Keywords: schizophrenia, quality of life, scale, assessment, Quality of Life Scale (QLS)

\footnotetext{
${ }^{1}$ Licenciada em Enfermagem pela Escola Superior de Saúde da Guarda, Especialista em Enfermagem de Saúde Mental e Psiquiatria, título concedido pela Ordem dos Enfermeiros, Mestre em Psicologia Clínica e da Saúde pela Universidade de Aveiro, Doutoranda em Psicologia na Universidade de Aveiro. Enfermeira numa Unidade de Cuidados na Comunidade do ACES Dão Lafões, Avenida Madre Rita de Jesus 3500-179 Viseu, Portugal. Tel.: +351916545031. E-mail: larapinho7@gmail.com

${ }^{2}$ Licenciada em Psicologia pela Faculdade de Psicologia e Ciências da Educação da Universidade de Coimbra, Doutorada em Psicologia (PhD) pela Universidade de Hull. Professora Associada com Agregação no Departamento de Educação e Psicologia, CIDTFF, CINTESIS, Universidade de Aveiro, Campus Universitário de Santiago, 3810-193 Aveiro, Portugal. Tel.: +351234370353 (ext. 24213). E-mail: anabelapereira@ua.pt

${ }^{3}$ Licenciada em Enfermagem, Especialista em Enfermagem de Saúde na Comunidade, título concedido pela Ordem dos Enfermeiros, Mestre em Síndrome de Imunodeficiência Adquirida: da Prevenção à Terapêutica, Doutorada em Ciências da Educação (PhD), Professora Associada na Escola Superior de Saúde de Viseu, IPV, CI\&DETS, R. D. João Crisóstomo Gomes de Almeida, n. ${ }^{\circ} 102$, 3500-843 Viseu, Portugal. Tel.: +351232419100 (ext. 7039). E-mail: cchaves@essv.ipv.pt
} 


\section{Introdução}

A esquizofrenia é, atualmente, considerada como uma doença mental grave e crónica que atinge cerca de $1 \%$ da população mundial. Em pleno século XXI e após mais de um século de estudos sobre esta patologia psiquiátrica, a sua causa permanece ainda desconhecida. Mesmo no que diz respeito ao tratamento farmacológico da doença, utilizado há mais de meio século, existe ainda pouca evidência de que produza resultados substancialmente positivos para a maioria das pessoas com esquizofrenia (Insel, 2010).

No que respeita à taxa de mortalidade, as pessoas com esquizofrenia têm 40 a $60 \%$ mais probabilidade de morte prematura do que a população geral, devido não só a problemas de saúde física, que são muitas vezes descorados, (como cancro, doenças cardiovasculares, diabetes e HIV) mas também à elevada taxa de suicídio nesta população (World Health Organization, 2013).

A esquizofrenia é definida pelo Manual de Diagnóstico e Estatística das Perturbações Mentais, versão 5, (DSM 5) da American Psychiatric Association, 2013, pela presença, num período mínimo de 6 meses, da seguinte sintomatologia: delírios, alucinações, sintomas negativos (diminuição da expressão emocional, avolição, alogia, anedonia, isolamento social), desorganização do pensamento e/ou comportamento. Esta constelação de sinais e sintomas está associada a uma série de disfunções cognitivas, comportamentais e emocionais, que conduzem, frequentemente, a um grave funcionamento social e ocupacional. Relativamente aos fatores sociais, a maioria dos doentes permanece solteiro, sem qualquer companheiro, e tem contatos sociais limitados fora do seu ambiente familiar (American Psychiatric Association, 2013). Os melhores preditores da deterioração no funcionamento social parecem ser os sintomas negativos (Couture, Granholm, \& Fish, 2011). Já no que concerne a fatores ocupacionais, a maioria dos doentes com diagnóstico de esquizofrenia estão desempregados, estando muitos deles incapacitados para o trabalho (Harvey et al., 2012). Além disto, a discriminação por parte da família, amigos, empregadores e desconhecidos é ainda hoje uma realidade (Gerlinger et al., 2013). Tendo em conta este panorama, a ansiedade é outro dos sintomas largamente presente nos indivíduos com esquizofrenia (Braga, Reynolds, \& Siris, 2013). Além da sintomatologia, todos estes fatores conduzem a uma baixa qualidade de vida nestes indivíduos.

A esquizofrenia é considerada uma das mais graves doenças mentais em termos de impacto negativo na qualidade de vida, não só nos doentes como também na família e na comunidade em que se inserem (Seeman, 2016). Estudos recentes demonstram a associação entre a sintomatologia da esquizofrenia e a baixa qualidade de vida nos indivíduos que dela padecem (Alessandrini et al., 2016; Gómez-de-Regil, 2015; Górna et al., 2014; Song et al., 2011). A qualidade de vida dos indivíduos com esquizofrenia depende não só da gravidade dos sintomas e da sua interferência com os seus objetivos de vida, como também com o número de interações sociais, dos fatores socioeconómicos, psicológicos, de saúde e do tratamento efetuado (Seeman, 2016).

A preocupação com a qualidade de vida dos indivíduos com doença mental, especialmente na esquizofrenia, surgiu com o processo de desinstitucionalização psiquiátrica, que ocorreu na década de 1960, passando estes indivíduos a viver na comunidade (Awad \& Lakshmi, 2016).

Neste sentido, o plano de ação para a saúde mental 2013-2020 da Organização Mundial de Saúde, define, como um dos seus objetivos principais fortalecer a investigação em saúde mental onde se incluem a obtenção de resultados em saúde relacionados com a qualidade de vida (World Health Organization, 2013).

Como consta nas guidelines para o tratamento biológico da esquizofrenia da World Federation of Societies of Biological Psychiatry, os objetivos principais da reabilitação psicossocial das pessoas com este diagnóstico, devem estar focados, não só na remissão da sintomatologia através de tratamento farmacológico e na melhoria do funcionamento social, mas também na otimização do bem-estar psicológico e da qualidade de vida do indivíduo. Acrescentam que, a fase de estabilização da esquizofrenia corresponde a um período prolongado de reabilitação psicossocial em que a sintomatologia está controlada farmacologicamente e o foco centra-se em 
melhorar o funcionamento social e ocupacional. Os principais objetivos durante esta fase são assegurar a remissão ou controlo da sintomatologia, melhorar o nível de funcionamento e a qualidade de vida, identificar atempadamente qualquer sinal de descompensação da sintomatologia e garantir o seu tratamento imediato e monitorizar os efeitos secundários da medicação (Hasan et al., 2013).

Perante estas recomendações, identificamos a qualidade de vida como conceito fundamental a ter em conta na reabilitação psicossocial da pessoa com esquizofrenia, sendo que esta deve ser estudada, definindo-se estratégias para que seja melhorada não só no indivíduo, mas também na família e na comunidade em que se insere.

Além disso, o aumento da expectativa de vida tem conduzido a uma tendência crescente na importância da qualidade de vida dos indivíduos e das comunidades (Pires, Silva, Ferreira, \& Fagulha, 2014) e a procura do significado de uma doença crónica envolve o repensar as prioridades para se conseguir obter os melhores benefícios da vida (Torres, Pereira, \& Monteiro, 2014).

A definição da Organização Mundial de Saúde sobre qualidade de vida mantem-se atualizada e tem sido atualmente utilizada em diversos estudos. Assim, entende-se por qualidade de vida a perceção da pessoa sobre a sua posição na vida, tendo em conta o contexto sociocultural e de valores em que se encontra inserido atendendo aos seus objetivos, expetativas, padrões e preocupações. É um conceito multidimensional, influenciado pela saúde mental e física, relações sociais, nível de independência, crenças pessoais e pelo meio envolvente (WHOQOL Group, 1995).

A qualidade de vida tornou-se, nas últimas décadas, fundamental, refletindo uma nova imagem da saúde, vista sob uma perspetiva biopsicossocial. $\mathrm{O}$ conceito tem sido aplicado no cuidado ao doente, nos estudos clínicos e na análise econômica da saúde. O elevado interesse na temática levou ao desenvolvimento instrumentos de avaliação da qualidade de vida e documentou claramente a baixa qualidade de vida das pessoas que sofrem esquizofrenia (Awad \& Lakshmi, 2016).

A Quality of Life Scale (QLS) desenvolvida por Heinrichs, Hanlon e Carpenter, 1984, é um dos instrumentos mais utilizados a nível internacional para avaliar especificamente a qualidade de vida destes indivíduos. Uma revisão da literatura entre janeiro de 2009 e dezembro de 2013 sobre escalas utilizadas na avaliação de doentes com esquizofrenia com um total de 432 estudos onde foram utilizadas 35 escalas diferentes, demonstrou que a QLS foi a escala de qualidade de vida específica para a esquizofrenia mais utilizada (Karow, Wittmann, Schöttle, Schäfer, \& Lambert, 2014).

Este instrumento avalia o funcionamento dos doentes com esquizofrenia tendo em conta os sintomas negativos da doença, independentemente da presença ou ausência de sintomatologia positiva. Fazem parte integrante do mesmo, a avaliação das experiências pessoais, da qualidade das relações interpessoais e da produtividade no papel ocupacional. É constituída por 21 itens e a sua administração demora em média 45 minutos, limitando a sua aplicabilidade na prática clínica. Para ultrapassar esta limitação foram desenvolvidas versões reduzidas da escala diminuindo o tempo de administração e com valores totais fiáveis e precisos (Bilker et al., 2003; Fervaha \& Remington, 2013; Ritsner, Kurs, Ratner, \& Gibel, 2005). No estudo realizado por Bilker et al., 2003, chegou-se à conclusão que uma versão reduzida da escala com 7 itens predizia a pontuação total de 21 itens com alta precisão, obtendo-se resultados fiáveis e com resultado total da escala com pontuações idênticas à escala original com 21 itens. Mais tarde, a investigação de Fervaha e Remington, 2013, veio corroborar estes resultados, utilizando uma amostra de 1430 participantes, para a mesma escala de 7 itens. Assim, ambos os estudos demonstraram a validade do instrumento, tendo obtido boas propriedades psicométricas, quando comparado com a escala original, podendo ser usado com fiabilidade. Dado que o tempo de aplicação é substancialmente inferior à escala com 21 itens, este instrumento pode ser introduzido em estudos de investigação que preconizem a avaliação da qualidade de vida na esquizofrenia. Este questionário torna-se também funcional e passível de ser utilizado na prática clínica, dado o tempo reduzido de aplicação, principalmente quando se querem avaliar resultados de intervenções na qualidade de vida dos utentes (Bilker et al., 2003; Fervaha \& Remington, 2013; 
Fervaha, Foussias, Siddiqui, Agid, \& Remington, 2014).

Assim, não havendo em Portugal nenhuma escala de avaliação da qualidade de vida das pessoas com esquizofrenia validada, e tendo em conta o vasto uso em diversos estudos mundiais e à fiabilidade da QLS, optou-se por adaptar e validar este instrumento, na sua versão reduzida. O objetivo deste estudo de investigação é, então, estudar as qualidades psicométricas de validade e confiabilidade da escala QLS-PT versão reduzida, adaptando-a para a população portuguesa com o diagnóstico de esquizofrenia.

\section{Metodologia}

Trata-se de um estudo quantitativo, transversal cujo objetivo é adaptar e validar a escala QLS abreviada, constituída por 7 itens, para a população portuguesa. $\mathrm{O}$ instrumento foi aplicado durante um período de 15 meses, entre janeiro de 2015 a março de 2016. A recolha de dados foi realizada em nove instituições de saúde de norte a sul de Portugal Continental nos serviços de ambulatório e/ou internamento de cinco Hospitais Psiquiátricos, três Departamentos de Psiquiatria de Hospitais Gerais e um Fórum Sócio-ocupacional.

A amostra foi do tipo não probabilístico, por conveniência, uma vez que foram convidados a participar no estudo todos os indivíduos que se encontravam disponíveis nos serviços referidos, tendo sido encaminhados pelo psiquiatra assistente, conforme os critérios de seleção.

A amostra foi, então, constituída por 282 utentes que se encontravam diagnosticados pelo psiquiatra, com esquizofrenia, segundo o critério da Classificação Internacional das Doenças (CID10) da World Health Organization, 2014.

Os critérios de elegibilidade dos participantes foram: terem contato com a comunidade, mesmo se institucionalizados; não apresentarem sintomatologia psicótica ativa que impedisse a compreensão dos objetivos da investigação, como delírios ou alucinações, e não apresentarem diagnóstico concomitante de oligofrenia. Estes critérios foram observados pelo psiquiatra assistente.

A recolha de dados foi efetuada em gabinete privado, individualmente, através de entrevista, conduzida pelo investigador, demorando entre 20 a 30 minutos.

No Quadro 1 podem observar-se as características gerais da amostra em estudo, sendo que as idades estão compreendidas entre 20 e 78 , sendo a média de $46,2(\sigma=13,13)$. A maioria é do sexo masculino $(60,3 \%)$, solteiro $(67,4 \%)$ e somente $14,2 \%$ se encontram casados. Relativamente à duração do diagnóstico, 50,7\% tem a doença há mais de 20 anos.

Quadro 1. Características gerais da amostra

\begin{tabular}{|c|c|c|}
\hline & \multicolumn{2}{|c|}{$\begin{array}{c}\text { Amostra } \\
(n=282)\end{array}$} \\
\hline & $\mathrm{N}$ & $\%$ \\
\hline \multicolumn{3}{|l|}{ Idade } \\
\hline Menos de 45 & 154 & 54,6 \\
\hline Mais de 45 & 128 & 45,4 \\
\hline \multicolumn{3}{|l|}{ Sexo } \\
\hline Masculino & 170 & 60,3 \\
\hline Feminino & 112 & 39,7 \\
\hline \multicolumn{3}{|l|}{ Habilitações Literárias } \\
\hline Sem escolaridade & 6 & 2,1 \\
\hline $1^{\circ}$ ciclo do Ensino Básico & 69 & 24,5 \\
\hline $2^{\circ}$ e $3^{\circ}$ ciclo do Ensino Básico & 132 & 46,9 \\
\hline Ensino Secundário $\left(12^{\circ}\right.$ ano $)$ & 54 & 19,1 \\
\hline Formação Universitária & 21 & 7,4 \\
\hline \multicolumn{3}{|l|}{ Estado Civil } \\
\hline Solteiro & 190 & 67,4 \\
\hline Casado / União de facto & 40 & 14,2 \\
\hline Divorciado / Separado & 44 & 15,6 \\
\hline Viúvo & 8 & 2,8 \\
\hline \multicolumn{3}{|l|}{ Duração do diagnóstico } \\
\hline$<5$ anos & 43 & 15,3 \\
\hline 5 a 10 anos & 35 & 12,4 \\
\hline 10 a 20 anos & 61 & 21,6 \\
\hline$>20$ anos & 143 & 50,7 \\
\hline
\end{tabular}

\section{Instrumentos de medida}

Foram utilizados dois instrumentos para medir a qualidade de vida.

\section{Escala QLSS $\mathbf{P T}$}

$\mathrm{O}$ instrumento de medida a ser validado é a Quality of Life Scale (QLS) de Heinrichs et al., 1984, na sua versão reduzida com 7 itens, validada nos estudos de Bilker et al., 2003 e, posteriormente, no estudo de Fervaha e Remington, 2013. Cada item é composto por uma escala do tipo Likert de sete pontos, sendo que valores de 5 e 6 indicam o funcionamento normal, de 2 a 4 revelam um considerável prejuízo e 0 e 1 refletem um prejuízo grave no item avaliado. Esta versão avalia as funções intrapsíquicas, as relações interpessoais, o papel ocupacional e os 
objetos comuns (Bilker et al., 2003; Fervaha \& Remington, 2013).

\section{Escala WHOQOL-BREF}

Esta escala foi elaborada por um grupo de investigadores da Organização Mundial de Saúde, em 1997, e trata-se de um instrumento genérico de avaliação da qualidade de vida que pode ser aplicado a pessoas saudáveis ou com doença. É constituída por um total de 26 itens que se dividem em quatro domínios: físico, psicológico, relações socias e meio ambiente. Cada item é composto por uma escala de Likert com 5 pontos. A escala foi validada para a população portuguesa, em 2007, por Canavarro e colaboradores.

\section{Procedimentos}

No decorrer do estudo foram respeitados todos os princípios éticos decorrentes de qualquer investigação, destacando-se o consentimento informado dos participantes, a confidencialidade e o anonimato e a autorização dos Conselhos de Administração das instituições onde decorreu o estudo. Além disso obteve-se parecer favorável da Comissão Nacional de Proteção de Dados (autorização $n^{\circ}$ 843/2015).

$\mathrm{Na}$ etapa de tradução seguiu-se a metodologia habitual, tendo-se obtido em primeiro lugar autorização para a tradução da escala e utilização em Portugal, pelos autores da mesma. Seguiu-se a tradução da escala QLS abreviada para português a partir da versão original em inglês, sendo posteriormente realizada a retroversão. Foram analisados os aspetos convergentes/divergentes com outros investigadores. Uma vez que a escala QLS se encontra validada em Espanha e no Brasil, foi efetuada uma análise comparativa das versões existentes nestes países, tendo em conta as semelhanças linguísticas e culturais. Foi realizada uma análise semântica da escala para que a mesma se adequasse à cultura portuguesa. Este processo foi facilitado devido ao facto não só de ser um instrumento para ser aplicado pelo entrevistador, como também ao facto de cada item ser composto por um conjunto de questões sugeridas a serem utilizadas e ajustadas ao nível de compreensão do entrevistado.

Após o processo de tradução, procedemos às análises estatísticas de validade e confiabilidade confiabilidade da escala.

\section{Análises estatísticas}

Segundo Laros (2005), a análise fatorial é imprescindível na validação de instrumentos psicológicos, podendo usar-se a análise fatorial exploratória ou a análise fatorial confirmatória. Neste estudo optou-se por utilizar a análise fatorial exploratória, uma vez que esta foi utilizada na validação da escala para outras populações, sendo que nem todas as versões são constituídas pelas mesmas subescalas. Assim, para aferir as propriedades psicométricas do instrumento, a estrutura relacional foi avaliada através da análise fatorial exploratória sobre a matriz das correlações, com extração dos fatores pelo método das componentes principais, seguida de uma rotação Varimax. Para avaliar a validade da análise fatorial exploratória utilizou-se o critério Kaiser-Meyer-Olkin (KMO) com os critérios de classificação definidos em Pereira e Patrício, 2013. A confiabilidade dos resultados da escala foi avaliada através da análise da consistência interna pelo cálculo do coeficiente Alfa de Cronbach. Foi ainda calculado o coeficiente de correlação de Pearson para verificar a correlação entre os fatores identificados e a escala global. Os dados foram analisados com o auxílio do IBM ${ }^{\circledR}$ SPSS $^{\circledR}$ Statistics, versão 22.0, uma vez que este é o software de análise de dados mais utilizado nas Ciências Sociais e Humanas (Marôco, 2014).

\section{Resultados}

Seguidamente serão apresentados os principais resultados das análises de validação da escala $\mathrm{QLS}_{7} \mathrm{PT}$, tendo em conta o objetivo do estudo: análise fatorial exploratória, validade concomitante, consistência interna e estatística descritiva.

\section{Análise Fatorial Exploratória}

Relativamente à dimensão da amostra, Crocker e Algina (1986, cit. in Laros, 2005), referem que o tamanho mínimo para realização de uma análise fatorial é de 10 sujeitos por variável, com um mínimo de amostra total de 100 sujeitos. Estes pressupostos são cumpridos, uma vez que o 
número de variáveis a ser estuado é de 7 e a amostra é constituída por 282 indivíduos.

Foi, então, realizada uma análise exploratória dos componentes principais da escala QLS versão reduzida, com o objetivo de determinar a estrutura fatorial. Para avaliar a validade da análise fatorial exploratória utilizou-se o critério KMO que foi indicativo de uma matriz aceitável à realização da análise em componentes principais $(\mathrm{KMO}=0,711)$ (Pereira \& Patrício, 2013). Realizou-se também o teste de esfericidade de Bartlett que foi significativo $\quad\left(X^{2}(21)=518.489, \quad p<.001\right)$, confirmando que as variáveis são correlacionáveis.

Procedeu-se à análise de componentes principais que permitiu identificar dois fatores que explicam 59,99 \% da variância total (para eigenvalues superiores a 1 ), sendo que o primeiro explica por si só $39,701 \%$ da variância total e o segundo 20,285\% (Quadro 2).

Quadro 2. Resultado da análise fatorial (componentes principais)

\begin{tabular}{|c|c|c|c|}
\hline \multirow[b]{2}{*}{ Componente } & \multicolumn{3}{|c|}{ Valores próprios iniciais } \\
\hline & Total & $\begin{array}{c}\% \text { de } \\
\text { variância }\end{array}$ & $\begin{array}{c}\% \\
\text { cumulativa }\end{array}$ \\
\hline 1 & 2,779 & 39,701 & 39,701 \\
\hline 2 & 1,420 & 20,285 & 59,986 \\
\hline 3 & 0,806 & 11,507 & 71,494 \\
\hline 4 & 0,731 & 10,440 & 81,934 \\
\hline 5 & 0,594 & 8,484 & 90,418 \\
\hline 6 & 0,353 & 5,050 & 95,468 \\
\hline 7 & 0,317 & 4,532 & 100,000 \\
\hline
\end{tabular}

Recorrendo à análise dos gráficos da variância pelo número de componentes (Scree Plot) verificamos que o ponto de inflexão da curva ocorre entre o $2^{\circ}$ e $3^{\circ}$ fator. Assim, o número mínimo de fatores a reter é 2 , além de que, o $3^{\circ}$ fator apresenta um eigenvalue já inferior a 1 (Figura 1).

Em concordância com o critério de KMO (regra do eigenvalue superior a 1) e com o critério do Scree plot, a estrutura relacional dos itens da QLS versão reduzida é explicada por dois fatores latentes.

A fim de facilitar a interpretação dos resultados da análise fatorial, simplificar a estrutura fatorial e possuir significado psicológico, procedeu-se ao método de rotação ortogonal

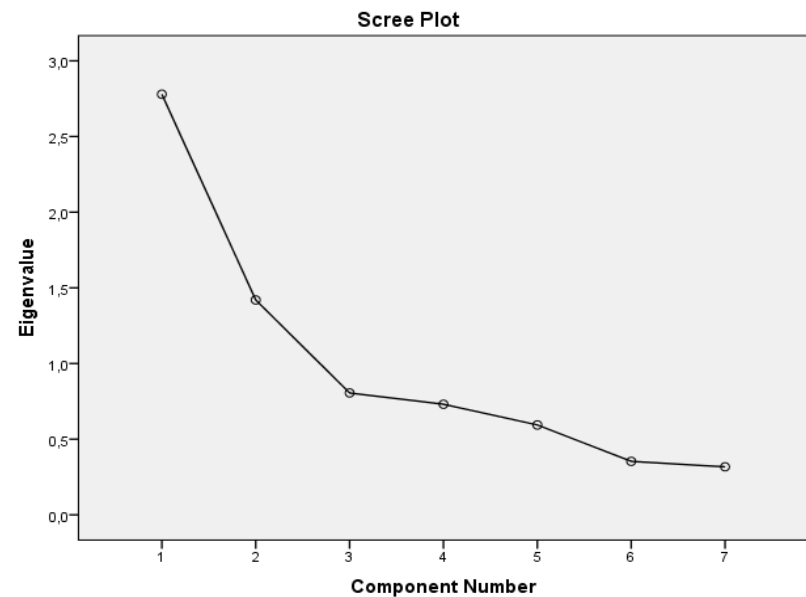

Figura 1. Scree Plot dos valores próprios

Varimax. O critério da rotação é, pois, maximizar a variância dos primeiros fatores, e em consequência desta rotação, maximizar a saturação de alguns itens. Utilizou-se a rotação ortogonal, uma vez que produz fatores que não se relacionam ou que são independentes uns dos outros.

O Quadro 3 mostra o peso fatorial de cada item nos 2 fatores retidos, eigenvalues e $\%$ da variância explicada, após uma Análise Fatorial Exploratória com extração de fatores pelo método das componentes principais, seguida de uma rotação Varimax. Apesar de os itens "relações ativas" e "iniciativas sociais" saturarem nos dois fatores, com cargas fatoriais superiores a .40 , muitos autores decidem reter o item com a carga fatorial mais elevada, sendo que outros referem ainda que a diferença entre os valores das cargas fatoriais deve exceder o valor de 0,20 (Pereira \& Patrício, 2013). De acordo com esta perspetiva, optou-se por manter os itens e considerar o item "relações ativas" no fator 1 (carga fatorial mais elevada 0,639$)$ e o item "iniciativas sociais" no fator 2 (carga fatorial mais elevada 0,712).

\section{Quadro 3. Estrutura fatorial}

\begin{tabular}{lcc}
\hline & Fator 1 & Fator 2 \\
\hline Relações ativas & $\mathbf{0 , 6 3 0}$ & 0,403 \\
Grau de motivação & $\mathbf{0 , 8 4 7}$ & $-0,024$ \\
Anedonia & $\mathbf{0 , 8 7 1}$ & 0,037 \\
Iniciativas Sociais & 0,411 & $\mathbf{0 , 7 1 2}$ \\
Funcionamento ocupacional & 0,392 & $\mathbf{0 , 4 5 9}$ \\
Objetos comuns & $-0,152$ & $\mathbf{0 , 7 9 9}$ \\
Capacidade de empatia & 0,076 & $\mathbf{0 , 6 7 4}$ \\
eigenvalues & 2,779 & 1,420 \\
Variância explicada & $39,701 \%$ & $20,285 \%$ \\
\hline
\end{tabular}


Tendo em conta estes resultados, a escala foi dividida em dois fatores. O primeiro fator contém 3 itens que correspondem aos componentes relações ativas, anedonia e grau de motivação. Optou-se por designar este fator por sintomas negativos e rede social uma vez que avalia dois sintomas negativos (anedonia e grau de motivação) e o relacionamento com pessoas conhecidas, sem envolvimento emocional íntimo (relações ativas). Pode-se observar que este fator tem uma boa consistência interna $(\alpha=.741)$. As correlações item/total variaram de .439 a .643 . O segundo fator contém 4 itens, iniciativas sociais, funcionamento ocupacional, objetos comuns e capacidade de empatia. Foi intitulado nível socio ocupacional uma vez que avalia a componente social com amigos e familiares (iniciativa social nas suas interações sociais e capacidade de empatia para com o outro) e avalia o funcionamento ocupacional e a posse de certos objetos básicos na sociedade em que se insere. A consistência interna deste fator foi aceitável $(\alpha=.650)$. As correlações item/total variaram de .371 a .534 (Pereira \& Patrício, 2013).

Pela análise destes resultados, verificamos que não se obteve uma replicação exata da estrutura fatorial da escala original.

Para verificar as correlações entre os fatores e o resultado global da QLS, foi realizada uma matriz de correlação (Quadro 4). Os resultados demostram que existem correlações fortes e significativas entre cada fator e a escala global (respetivamente, $r=.802 ; r=.887$ ) comparativamente às correlações obtidas entre os dois fatores $(r=.435)$, o que indica a presença de um constructo comum subjacente aos fatores, relacionado com o total da escala de qualidade de vida.

Quadro 4. Matriz de correlação entre fatores e resultado total da escala QLS

\begin{tabular}{|c|c|c|c|}
\hline & fator 1 & fator 2 & QLStotal \\
\hline \multicolumn{4}{|l|}{ Fator 1} \\
\hline Fator 2 & $0,435^{* *}$ & & \\
\hline QLStotal & $0,802^{* *}$ & $0,887^{* *}$ & \\
\hline
\end{tabular}

\section{Validade concomitante}

Foi avaliada a validade concomitante da escala comparando os resultados totais da QLS versão reduzida com os resultados da WHOQOLBREF, tendo-se obtido uma correlação significativa $(r=.498 ; p<.001)$.

\section{Consistência interna}

A escala apresentou uma boa consistência interna que foi avaliada através do alfa de Cronbach $(\alpha=.74)$. Relativamente ao fator 1 , composto por três questões, obteve-se uma boa consistência interna $(\alpha=.71)$. Já no que respeita o ao fator 2 , composto por quatro questões, a consistência interna foi aceitável $(\alpha=.63)$ (Pereira \& Patrício, 2013). Foram avaliadas as correlações de Pearson entre a escala total e os itens da escala e entre os fatores e os itens. As correlações item/escala total, item/fator 1 e item/fator 2 foram todas significativas $(p<.001)$.

As correlações item/escala total variaram entre $r=.437$ e $r=.778$. O item "iniciativas sociais" foi o que obteve uma correlação mais forte $(r=.778)$.

As correlações item/fator 1 variaram entre $r=.788$ e $r=.838$, sendo o item "anedonia" o que obteve correlação mais forte $(r=.838)$.

Já no que diz respeito ao fator 2 , as correlações variaram entre $r=.605$ e $r=.783$, sendo o fator com maior correlação "iniciativas sociais".

\section{Estatística descritiva}

As estatísticas descritivas dos itens da escala QLS $_{7}$ PT encontram-se discriminadas no quadro 5. Os valores médios dos itens da escala variaram entre 2,67 e 4,68, com desvio padrão entre 1,324 e 2,258 . Os valores mais altos foram obtidos nos itens grau de motivação, anedonia e capacidade de empatia. Relativamente ao valor total da escala este foi determinado através da média dos 7 itens, obtendo-se um valor de 3,84 , com desvio padrão de 1,040, revelando um considerável prejuízo na qualidade de vida destes doentes uma vez que se encontra entre 2 e 4 . Foram ainda realizadas análises descritivas para avaliar o número de indivíduos com funcionamento normal, com prejuízo considerável na qualidade de vida e com prejuízo grave (Quadro 6). Verifica-se que a maior parte da amostra $(70,57 \%)$ obteve uma pontuação total da escala $\mathrm{QLS}_{7} \mathrm{PT}$ entre 2 e 4 , existindo, deste modo, um prejuízo considerável na qualidade de vida. No entanto, apenas $1,06 \%$ obteve um prejuízo grave. 
Quadro 5. Estatísticas descritivas dos itens da escala $\mathrm{QLS}_{7}-\mathrm{PT}$

Mínimo Máximo Média Desvio Padrão

\begin{tabular}{lllll}
\hline 1.Relações ativas & 0 & 6 & 3,67 & 1,883 \\
2.Iniciativas Sociais & 0 & 6 & 2,84 & 1,882 \\
3.Funcionamento ocupacional & 0 & 6 & 2,67 & 2,258 \\
4.Grau de motivação & 1 & 6 & 4,65 & 1,324 \\
5.Anedonia & 0 & 6 & 4,68 & 1,416 \\
6.Objetos comuns & 1 & 6 & 3,69 & 1,369 \\
7.Capacidade de empatia & 0 & 6 & 4,66 & 1,359 \\
QLStotal & & & 3.84 & 1.040 \\
\hline
\end{tabular}

Quadro 6. Valores totais da $\mathrm{QLS}_{7} \mathrm{PT}$

\begin{tabular}{lcc}
\hline & $\mathrm{N}$ & $\%$ \\
\hline Funcionamento normal (5 e 6) & 80 & 28,37 \\
Prejuízo considerável (2 a 4) & 199 & 70,57 \\
Prejuízo grave (0 e 1) & 3 & 1,06 \\
\hline
\end{tabular}

\section{Discussão}

A esquizofrenia, devido à sua sintomatologia característica, está associada a disfunções sociais e ocupacionais que acometem a qualidade de vida. Esta talvez seja a patologia psiquiátrica que mostra de forma mais evidente o perfil de cronicidade e a baixa resposta às intervenções terapêuticas, evidenciando uma baixa qualidade de vida, confirmada por vários estudos de investigação (Cardoso et al., 2006; Cesari \& Bandeira, 2010; Song et al., 2011). No entanto, a avaliação da qualidade de vida dos doentes com diagnóstico de esquizofrenia não parece ser uma prática clínica habitual, o que se deve, provavelmente, à falta de instrumentos disponíveis específicos e à falta de perceção dos profissionais de saúde de que a deterioração do doente possa afetar a sua qualidade de vida (Suárez, Sánchez, \& Calvo, 2013).

A QLS é uma das escalas mais utilizadas a nível internacional para avaliar a qualidade de vida desta população, tendo boas propriedades psicométricas. A sua versão reduzida ao demorar menos tempo na aplicação torna-a passível de ser utilizada na prática clínica (Bilker et al., 2003; Fervaha \& Remington, 2013).

Os passos metodológicos utilizados para a adaptação transcultural deste instrumento foram essenciais para a adequação ao contexto português. À semelhança da versão inglesa da QLS reduzida (Bilker et al., 2003; Fervaha \&
Remington, 2013), também esta versão demonstrou possuir propriedades psicométricas adequadas de validade e confiabilidade para avaliar a qualidade de vida de pessoas com diagnóstico de esquizofrenia, em Portugal.

A análise fatorial da escala $\mathrm{QLS}_{7} \mathrm{PT}$ mostrou a distribuição dos 7 itens em dois fatores, explicando quase $60 \%$ da variância. Os dois fatores encontrados visam avaliar dimensões importantes na esquizofrenia, sendo que no primeiro fator se avaliam os sintomas negativos e a rede social e no segundo o nível ocupacional. Estes resultados diferem do estudo de validação da escala original de Heinrichs et al., 1984 que propõe uma distribuição da escala em quatro fatores (funções intrapsíquicas, relações interpessoais, papel ocupacional e objetos comuns e atividades). Já o estudo de validação da escala para Espanha (Rodríguez, Soler, Rodríguez, Esparcia, \& Miarons, 1995) propõe na sua análise fatorial uma distribuição por três fatores (funções intrapsíquicas, relações interpessoais e papel ocupacional) e o de validação da escala para o Brasil que propõe igualmente três fatores: rede social, nível ocupacional e funções intrapsíquicas e relações interpessoais, tendo sido eliminados dois itens (anedonia e grau de motivação).

No que respeita à consistência interna, habitualmente, considera-se que um instrumento tem fiabilidade aceitável quando o alfa de Cronbach é de pelo menos .60. Neste estudo, o valor de $\alpha$ da escala global foi de .74, demonstrando boa consistência interna. Relativamente aos dois fatores, o valor de $\alpha$ situase entre .65 e .74 o que indica uma adequada homogeneidade do instrumento (Pereira \& Patrício, 2013). 
Verificou-se ainda que ambos os fatores apresentaram coeficientes de correlação significativos com a escala global. Este resultado é esperado na validação de escalas e indica que os fatores do instrumento estão ligados a um constructo comum, que neste caso se refere à qualidade de vida dos doentes com esquizofrenia.

Assim, neste estudo, a média da QLS 7 PT total foi de 3.84 , sendo que, para a maior parte dos indivíduos (70,57\%) se situa entre 2 a 4, sugerindo um prejuízo bastante significativo da qualidade de vida para a maioria dos itens avaliados. Estes dados assemelham-se aos de validação da escala original com 21 itens de Heinrichs et al., 1984 e ao estudo posterior para a população brasileira de Cardoso et al., 2003, bem como ao estudo da versão reduzida com 7 itens de Fervaha e Remington, 2013, que também encontraram valores entre 2 e 4 para o total da escala QLS. Não obstante, esta investigação foi a que obteve uma média superior do total da escala. Estes resultados revelam o impacto da doença na qualidade de vida.

$\mathrm{Na}$ presente investigação, os resultados mais baixos da $\mathrm{QLS}_{7} \mathrm{PT}$ referem-se aos itens iniciativas sociais e funcionamento ocupacional, seguidos das relações ativas, comprovando-se um prejuízo ao nível sócio ocupacional das pessoas com o diagnóstico de esquizofrenia. Neste sentido, consideramos ser necessária uma intervenção eficaz e eficiente dos profissionais de saúde mental na reabilitação psicossocial das pessoas com o diagnóstico de esquizofrenia, promovendo atividades sociais e ocupacionais.

Tendo em conta os resultados globais, verificámos que os dados corroboram os dos estudos de Bilker et al, 2003 e de Fervaha e Remington, 2013 quando referem que a QLS versão reduzida com 7 itens pode ser utilizada com grande precisão. Cabe-nos concluir que, os resultados do presente estudo mostram que a escala $\mathrm{QLS}_{7} \mathrm{PT}$ tem características psicométricas adequadas, podendo ser utilizada para a população portuguesa. Este instrumento torna-se assim de grande utilidade para a prática clinica uma vez que, através da avaliação dos défices apresentados a nível social e ocupacional destes doentes, poderão ser definidas estratégias para a reabilitação psicossocial, que após a desinstitucionalização psiquiátrica dos doentes mentais obteve um papel fundamental da reinserção social dos utentes com esquizofrenia.

\section{Referências}

Alessandrini, M., Lançon, C., Fond, G., FagetAgius, C., Richieri, R., Faugere, M.,... Boyer, L. (2016). A structural equation modelling approach to explore the determinants of quality of life in schizophrenia. Schizophrenia research, 171(1), 27-34. doi: 10.1016/j.schres.2016.01.012

American Psychiatric Association (2013). Diagnostic and Statistical Manual of Mental Disorders (DSM-5). Fifth Edition. Washington, DC: American Psychiatric Pub.

Awad, A. G., \& Voruganti, L. N. (2016). Beyond Assessment of Quality of Life in Schizophrenia. Switzerland: Adis. ISBN: 9783-319-30061-0

Bilker, W. B., Brensinger, C., Kurtz, M. M., Kohler, C., Gur, R. C., Siegel, S. J., \& Gur, R. E. (2003). Development of an abbreviated schizophrenia quality of life scale using a new method. Neuropsychopharmacology: official publication of the American College of Neuropsychopharmacology, 28(4), 773-777. doi: 10.1038/sj.npp.1300093

Braga, R. J., Reynolds, G. P., \& Siris, S. G. (2013). Anxiety comorbidity in schizophrenia. Psychiatry research, 210(1), 17. doi: 10.1016/j.psychres.2013.07.030

Canavarro, M. C., Simões, M. R., Vaz Serra, A., Pereira, M., Rijo, D., Quartilho M. J.,... Carona, C. (2007). WHOQOL-Bref: Instrumento de avaliação da qualidade de vida da Organização Mundial de Saúde, In: M. R. Simões, C. Machado, M. M. Gonçalves \& L. S. Almeida (Coords). Avaliação psicológica: Instrumentos validados para a população portuguesa. (pp. 77-100). Coimbra: Quarteto Editora.

Cardoso, C. S., Caiaffa, W. T., Bandeira, M., Siqueira, A. L., Fonseca, I. K., \& Fonseca, J. O. P. (2003). Qualidades psicométricas da escala de qualidade de vida para pacientes com esquizofrenia: Escala QLS-BR. Jornal Brasileiro de Psiquiatria, 52(3), 211-22.

Cardoso, C. S., Caiaffa, W. T., Bandeira, M., Siqueira, A. L., Abreu, M. N. S., \& Fonseca, 
J. O. P. (2006). Qualidade de vida e dimensão ocupacional na esquizofrenia: Uma comparação por sexo. Cadernos de Saúde Pública, 22(6), 1303-1314.

Cesari, L., \& Bandeira, M. (2010). Avaliação da qualidade de vida e percepção de mudança em pacientes com esquizofrenia. Jornal Brasileiro de Psiquiatria, 59(4), 293-301.

Couture, S. M., Granholm, E. L., \& Fish, S. C. (2011). A path model investigation of neurocognition, theory of mind, social competence, negative symptoms and realworld functioning in schizophrenia. Schizophrenia Research, 125 (2-3) 152-160. doi:10.1016/j.schres.2010.09.020.

Fervaha, G., \& Remington, G. (2013). Validation of an abbreviated quality of life scale for schizophrenia.

European Neuropsychopharmacology, 23(9), 1072-1077.

Fervaha, G., Foussias, G., Siddiqui, I., Agid, O., \& Remington, G. (2014). Abbreviated quality of life scales for schizophrenia: Comparison and utility of two brief community functioning measures. Schizophrenia Research, 154(1), 8992.

Gerlinger, G., Hauser, M., Hert, M., Lacluyse, K., Wampers, M., \& Correll, C. U. (2013). Personal stigma in schizophrenia spectrum disorders: A systematic review of prevalence rates, correlates, impact and interventions. World Psychiatry, 12(2), 155164.

Gómez-de-Regil, L. (2015). Curso de la enfermedad y calidad de vida en pacientes mexicanos con psicosis. Revista de Psiquiatría y Salud Mental, 8(4), 218-223. doi: 10.1016/j.rpsm.2013.12.001

Górna, K., Jaracz, K., Jaracz, J., Kiejda, J., Grabowska-Fudala, B., \& Rybakowski, J. (2014). Social functioning and quality of life in schizophrenia patients: Relationship with symptomatic remission and duration of illness. Psychiatria polska, 48(2), 277-288.

Hasan, A., Falkai, P., Wobrock, T., Lieberman, J., Glenthoj, B., Gattaz, W. F.,... Möller, H. J. (2013). World Federation of Societies of Biological Psychiatry (WFSBP) guidelines for Biological treatment of schizophrenia, part 2: Update 2012 on the long-term treatment of schizophrenia and management of antipsychotic-induced side effects. The World Journal of Biological Psychiatry, 14(1), 2-44. doi: 10.3109/15622975.2012.739708

Harvey, P. D., Heaton, R. K., Carpenter, W. T., Green, M. F., Gold, J. M., \& Schoenbaum, M. (2012). Functional impairment in people with schizophrenia: Focus on employability and eligibility for disability compensation. Schizophrenia research, 140(1), 1-8. doi: 10.1016/j.schres.201 2.03.025.

Heinrichs, D. W., Hanlon, T. E., \& Carpenter, W. T. (1984). The quality of life scale: An instrument for rating the schizophrenic deficit syndrome. Schizophrenia Bulletin, 10(3) 388398.

Insel, T. R. (2010). Rethinking schizophrenia. Nature, 468(7321) 187-193. doi: 10.1038/nature09552

Karow, A., Wittmann, L., Schöttle, D., Schäfer, I., \& Lambert, M. (2014). The assessment of quality of life in clinical practice in patients with schizophrenia. Dialogues in clinical neuroscience, 16(2), 185.

Laros, J. A. (2005). O uso da análise fatorial: algumas diretrizes para pesquisadores. In: $\mathrm{L}$. Pasquali (Org.). Análise fatorial para pesquisadores. (pp. 147-170). Brasília: Editora da Universidade de Brasília.

Marôco, J. (2014). Análise Estatística com o SPSS Statistics. $6^{\mathrm{a}}$ ed. Pêro Pinheiro: ReportNumber. ISBN: 978-989-96763-4-3

Pereira, A., \& Patrício, T. (2013). SPSS - Guia prático de utilização - análise de dados para ciências sociais e psicologia. $8^{\mathrm{a}} \mathrm{ed}$. Lisboa: Edições Sílabo. ISBN: 978-972-618-736-3.

Pires, R., Silva, D. R., Ferreira, A. S., \& Fagulha, T. (2014). Relações entre a qualidade de vida e os estilos de personalidade medidos com a adaptação Portuguesa do Índice de Estilos da Personalidade de Millon - MIPS-R. Revista Iberoamericana de Diagnóstico y Evaluación - e Avaliação Psicológica, 37(1), 53-72.

Ritsner, M., Kurs, R., Ratner, Y., \& Gibel, A. (2005). Condensed version of the Quality of Life Scale for schizophrenia for use in outcome studies. Psychiatry research, 135(1), 65-75. doi: 10.1016/j.psychres.2005.01.007

Rodríguez, A., Soler, R. M., Rodríguez, A., Esparcia, A. J. J., \& Miarons, R. (1995). Estudio factorial y adaptación de la escala de calidad de vida en la esquizofrenia 
(QLS). Revista de psicología general y aplicada: Revista de la Federación Española de Asociaciones de Psicología, 48(3), 353364.

Seeman, M. (2016). Schizophrenia and Its Sequelae. In A. G. Awad \& L. N. Voruganti (Eds), Beyond Assessment of Quality of Life in Schizophrenia. (pp. 3-14). Switzerland: Adis. ISBN: 978-3-319-30061-0

Song, Y. Y., Kim, K. R., Park, J. Y., Lee, S. Y., Kang, J. I., Lee, E., ... Kwon, J. S. (2011). Associated factors of quality of life in firstepisode schizophrenia patients. Psychiatry investigation, 8(3), 201-206. doi: 10.4306/pi.2 011.8.3.201

Suárez, M. F., Sánchez, R., \& Calvo, J. M. (2013). Validación de la escala Schizophrenia Quality Of Life Scale (SQLS) para medir la calidad de vida en pacientes con diagnóstico de esquizofrenia en Colombia. Revista Colombiana de Psiquiatría, 42(3), 257-265. doi: 10.1016/S0034-7450(13)70018-0

Torres, A., Pereira, A., \& Monteiro, S. (2014). Validity study of Portuguese version of Cancer Coping Questionnaire. Revista Iberoamericana de Diagnóstico y Evaluación - e Avaliação Psicológica, 2(38), 199-220.

WHOQOL Group (1995). The World Health Organization Quality of Life Assessment (WHOQOL): Position paper from the World Health Organization. Social Science $e$ Medicine, 41(10), 1403-1409.

World Health Organization (2013). Comprehensive mental health action plan 2013-2020. Geneva: Author.

World Health Organization (2014). International Statistical Classification of Diseases and Related Health Problems, 10th Revision (ICD-10). Disponível em: http://apps.who.int/ classifications/icd10/browse/2014/en\#/ 